ARTICLE

https://doi.org/10.1038/s41467-018-07876-6

\title{
Chromosome conformation capture resolved near complete genome assembly of broomcorn millet
}

Junpeng Shi (1) ${ }^{1}$, Xuxu Ma', Jihong Zhang ${ }^{1}$, Yingsi Zhou', Minxuan Liu², Liangliang Huang ${ }^{1}$, Silong Sun (1) 1 , Xiangbo Zhang ${ }^{1}$, Xiang Gao', Wei Zhan ${ }^{3}$, Pinghua Li ${ }^{4}$, Lun Wang ${ }^{5}$, Ping Lu², Haiming Zhao', Weibin Song ${ }^{1} \&$ Jinsheng Lai (iD) ${ }^{1,6}$

Broomcorn millet (Panicum miliaceum L.) has strong tolerance to abiotic stresses, and is probably one of the oldest crops, with its earliest cultivation that dated back to ca. 10,000 years. We report here its genome assembly through a combination of PacBio sequencing, BioNano, and Hi-C (in vivo) mapping. The 18 super scaffolds cover $~ 95.6 \%$ of the estimated genome $(\sim 887.8 \mathrm{Mb})$. There are 63,671 protein-coding genes annotated in this tetraploid genome. About $\sim 86.2 \%$ of the syntenic genes in foxtail millet have two homologous copies in broomcorn millet, indicating rare gene loss after tetraploidization in broomcorn millet. Phylogenetic analysis reveals that broomcorn millet and foxtail millet diverged around $\sim 13.1$ Million years ago (Mya), while the lineage specific tetraploidization of broomcorn millet may be happened within $\sim 5.91$ million years. The genome is not only beneficial for the genome assisted breeding of broomcorn millet, but also an important resource for other Panicum species.

\footnotetext{
${ }^{1}$ State Key Laboratory of Agrobiotechnology and National Maize Improvement Center, Department of Plant Genetics and Breeding, China Agricultural University, Beijing 100193, P. R. China. ${ }^{2}$ Institute of Crop Science, Chinese Academy of Agricultural Sciences, Beijing 100081, P. R. China. ${ }^{3}$ Annoroad Gene Technology (Beijing) Co., Ltd, Beijing 100176, P. R. China. ${ }^{4}$ State Key Laboratory of Crop Biology, College of Agronomy, Shandong Agricultural University, Tai'an 271018, P. R. China. ${ }^{5}$ Institute of Crop Germplasm Resources, Shanxi Academy of Agricultural Sciences, Taiyuan 030031, P. R. China. ${ }^{6}$ Center for Crop Functional Genomics and Molecular Breeding, China Agricultural University, Beijing 100193, P. R. China. These authors contributed equally: Junpeng Shi, Xuxu Ma, Jihong Zhang. Correspondence and requests for materials should be addressed to W.S. (email: songweibin@cau.edu.cn)

or to J.L. (email: jlai@cau.edu.cn)
} 
M illets are a variety of small-seeded grass that are widely grown, particularly in resource-poor areas around Asia and Africa ${ }^{1}$. Broomcorn millet (Panicum miliaceum L.), also known as common millet or proso millet, is probably one of the oldest crops around the world, with its origin from northern China that could be dated back to $c a$. $~ 10,000$ years before present $(\mathrm{cal} \text { yr BP })^{2}$. Archeological evidence also suggested another possible origin center of broomcorn millet in eastern Europe, with its earliest cultivation that occurred $\sim 7000 \mathrm{cal} \mathrm{yr} \mathrm{BP}^{3,4}$. Broomcorn millet was a recent allotetraploid $(2 \mathrm{n}=4 \times=36)$ with its two subgenomes originated from two species closely related to $P$. capillare and $P$. repens ${ }^{3,5}$. It could be used as a pioneer crop at marginal regions due to its short growing season (reaching maturity after $\sim 60-90$ days), extremely low water requirements, high salt tolerance, and nutrient resource usage efficiency ${ }^{6,7}$. However, the grain yield of broomcorn millet is relatively low when compared with its close relative foxtail millet, which may be partially attributed to its little genetic gains up to now. As a demonstration of the feasibility of using $10 \mathrm{X}$ linked reads to assemble highly complex crop genomes, a draft genome sequence of broomcorn millet has been recently released ${ }^{5}$. However, no high-quality reference genome has been reported in the entire Panicum genus, which includes other important species, such as switchgrass (Panicum virgatum L.) ${ }^{8}$.

The genomes of crop plants often have undergone polyploidization and have relatively high proportion of repeat elements (especially LTR retrotransposons) $)^{9,10}$. Despite the prosperity of assembling crop genomes due to the application of Illumina sequencing technologies, the majority of assemblies were remained to be in draft status due to the difficulty of assembling short Illumina reads ${ }^{11}$. Owing to the advent of SMRT (Single Molecule Real-Time) sequencing technologies, especially the popularization of PacBio sequencing which could generate reads up to $\sim 30-40 \mathrm{~kb}$, the continuity of genome assemblies (such as rice $^{12,13}$, maize $e^{14,15}$, Aegilops tauschii ${ }^{16,17}$, durian ${ }^{18}$, and quinoa $^{19,20}$ ) reached several orders of magnitude higher (N50 $>1$ $\mathrm{Mb}$ ) when compared with Illumina assemblies (usually tens of kilobases). In addition, the combination of BioNano optical mapping and chromosome conformation information, generated by either in vivo (Hi-C) ${ }^{18,21}$ or in vitro (Chicago) ${ }^{19}$ technologies, was shown to be able to anchor the scaffolds into chromosomal or subchromosomal levels.

Here, we report a high-quality de novo genome assembly of broomcorn millet. Through a combination of PacBio sequencing, BioNano optical mapping and Hi-C (in vivo) mapping, we generate a chromosome scale assembly with a total scaffold length of $\sim 848.4 \mathrm{Mb}$ ( $\mathrm{N} 50$ of $\sim 8.24 \mathrm{Mb}$ ) that accounted for $\sim 95.6 \%$ of the estimated genome size ( $\sim 887.8 \mathrm{Mb})$. We annotate 63,671 genes in broomcorn millet, which is nearly two times the number of genes $(34,584)$ in foxtail millet. There are 19,609 genes in foxtail millet that are syntenic with broomcorn millet, among which 16,884 ( $86.2 \%)$ genes have two homologous copies retained in broomcorn millet, indicating rare gene loss after whole-genome duplication (WGD). Phylogenetic analysis reveals a common ancestor before 13.1 million years ago (Mya) between broomcorn millet and foxtail millet, and the tetraploidization is estimated to be happened within $\sim 5.91$ million years. The genome sequence we report here is not only important to understand the dynamic evolution following genome tetraploidization in Paniceae, but also benefit the molecular breeding of broomcorn millet in the future.

\section{Results}

Genome sequencing and assembly of broomcorn millet. To sequence the genome of broomcorn millet, we selected an elite cultivar named Longmi4 (Supplementary Fig. 1), which was widely cultivated among the northern region of China. We first generated $\sim 103.4 \mathrm{~Gb}$ Illumina paired-end reads $(150 \mathrm{bp})$ to analyze the genome of Longmi4, which was highly homozygous (the heterozygosity ratio was $\sim 0.04 \%)^{22}$. The genome size was estimated to be $\sim 887.8 \mathrm{Mb}$ from k-mer analysis (Supplementary Fig. 2 and Supplementary Note 1). It was about two times the size of foxtail millet $(\sim 485 \mathrm{Mb})^{23,24}$, which was consistent with previous assumption that a tetraploidization event happened in the lineage of broomcorn millet after its divergence with Setaria ${ }^{23}$. While, there was a large difference of genome size between broomcorn millet and switchgrass $(\sim 1220.0 \mathrm{Mb}$, Panicum virgatum v1.0, DOE-JGI, http://phytozome.jgi.doe.gov/), since two independent polyploidization events were inferred to be happened in these two species ${ }^{23}$.

We totally generated $\sim 150.7 \mathrm{~Gb}$ subreads $(\mathrm{N} 50=12.6 \mathrm{~kb})$ from PacBio Sequel platform, which covered $\sim 170 \mathrm{x}$ of Longmi4 genome (Supplementary Table 1). Falcon ${ }^{25}$ was firstly used to self-correct and assemble the PacBio reads, then polished with both PacBio and Illumina reads $(\sim 116 \times)$ to generate 1262 consensus contigs $(\sim 839.0 \mathrm{Mb})$ with contig N50 of $\sim 2.55 \mathrm{Mb}$ (Supplementary Note 2). We further generated BioNano optical maps $(\sim 235 \times, \mathrm{N} 50=$ $255.2 \mathrm{~kb}$, Supplementary Table 2), resolved the conflicts in original contigs into 1308 contigs and anchored these contigs into 905 scaffolds $(\sim 848.4 \mathrm{Mb})$ with scaffold N50 of $\sim 8.24 \mathrm{Mb}$ (Table 1). About $\sim 95.6 \%$ of the estimated genome was covered by the scaffolds, with 127 longest scaffolds that accounted for more than $90 \%$ of the genome $(\mathrm{N} 90=1.47 \mathrm{Mb})$. To evaluate the assembly quality, we mapped both the Illumina and RNA-seq reads back to the scaffolds, with mapping efficiencies of $\sim 99.6 \%$ and $\sim 91.5 \%$, respectively (Supplementary Table 3 ). We also evaluated the assembly with 1440 Benchmarking Universal Single Copy Orthologs (BUSCO) genes from embryophyta ${ }^{26}$, of which 1417 genes $(\sim 98.4 \%)$ were annotated and 1411 genes $(\sim 98.0 \%)$ were intact.

To further anchor and orient the scaffolds into super scaffolds, we constructed Hi-C (in vivo cross-linking of chromatins) libraries from the seedlings and generated $\sim 622.2$ million paired-end reads covering $\sim 140.2 \mathrm{x}$ of Longmi4 genome (Supplementary Table 4). As expected, the spatial proximity, as reflected by the Hi-C interaction intensity, decreased along with

\begin{tabular}{|c|c|c|c|}
\hline & Contigs & Scaffolds & $\begin{array}{l}\text { Super } \\
\text { scaffolds }\end{array}$ \\
\hline \multicolumn{4}{|l|}{ Assembly features } \\
\hline Numbers & 1308 & 905 & 475 \\
\hline Total length & $\sim 838.9 \mathrm{Mb}$ & $\sim 848.4 \mathrm{Mb}$ & $\sim 848.4 \mathrm{Mb}$ \\
\hline N50 & $\sim 2.55 \mathrm{Mb}$ & $\sim 8.24 \mathrm{Mb}$ & $\sim 48.26 \mathrm{Mb}$ \\
\hline Longest & $19,200,716$ bp & $22,633,379 b p$ & $69,183,459$ bp \\
\hline Coverage & $\sim 94.5 \%$ & $\sim 95.6 \%$ & $\sim 95.6 \%$ \\
\hline GC content & 46.80\% & & \\
\hline \multicolumn{4}{|l|}{ Protein-coding genes } \\
\hline $\begin{array}{l}\text { Numbers of } \\
\text { genes }\end{array}$ & 63,671 & & \\
\hline $\begin{array}{l}\text { Gene space } \\
\text { (coverage) }\end{array}$ & $\begin{array}{l}214,072,228 \mathrm{bp} \\
(\sim 24.1 \%)\end{array}$ & & \\
\hline $\begin{array}{l}\text { Number of } \\
\text { transcripts }\end{array}$ & 86,387 & & \\
\hline $\begin{array}{l}\text { Mean transcript } \\
\text { length }\end{array}$ & 2883 bp & & \\
\hline Mean CDS length & 1023 bp & & \\
\hline $\begin{array}{l}\text { Mean intron } \\
\text { length }\end{array}$ & 1270 bp & & \\
\hline
\end{tabular}




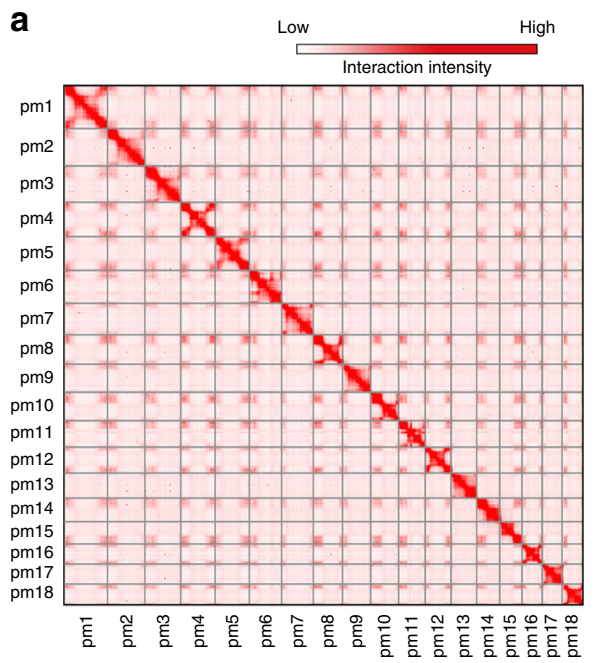

\section{b}

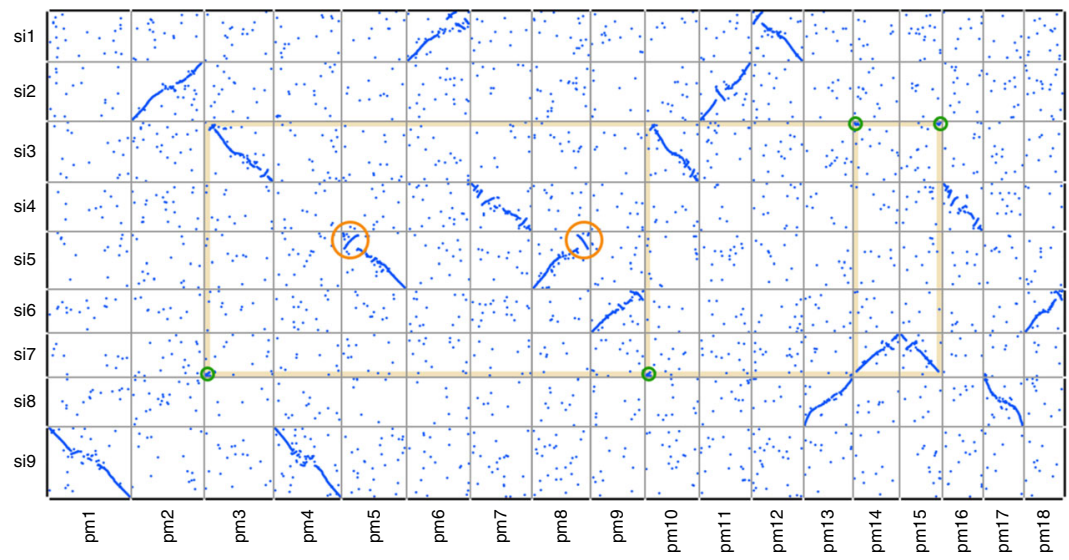

Fig. 1 The $\mathrm{Hi}-\mathrm{C}$ assisted assembly of Longmi4 pseudomolecules. a Heatmap showing $\mathrm{Hi}-\mathrm{C}$ interactions under the resolution of $200 \mathrm{~kb}$, and the antidiagonal pattern for the intrachromosomal interactions may reflect the Rabl configuration of chromatins. $\mathbf{b}$ Genome comparison between broomcorn millet (pm1-pm18) and foxtail millet (si1-si9). Each dot represented a homologous sequence reported by Mummer, and the two orange circles referred to two intrachromosomal inversions. The green circles represented the quartet of interchromosomal exchanges that may be happened before tetraploidization. Source Data of Fig. 1b are provided as a Source Data file

the increasing of physical distance between two loci (Supplementary Fig. 3). Therefore, we were able to cluster and orient 444 scaffolds ( $838.9 \mathrm{Mb}, \sim 98.9 \%)$ into 18 long super scaffolds (hereafter denoted as pseudomolecules, N50 $=48.3 \mathrm{Mb}$ ) through a hierarchical clustering strategy ${ }^{27}$. Upon the pseudomolecules we have constructed (Supplementary Table 5), the Hi-C interaction matrices displayed a distinct anti-diagonal pattern for the intrachromosomal interactions (Fig. 1a), which may reflect the so-called Rabl configuration that the long and short arms of chromatins folded parallelly in the interphase nuclei ${ }^{21,28}$.

Comparative genomic analysis revealed a 2-to-1 syntenic relationship between broomcorn millet and foxtail millet (Fig. 1b). Unlike maize which experienced strong chromosome fusions after tetraploidization ${ }^{29}$, the two sets of chromosomes were nearly intact in broomcorn millet. However, intrachromosomal rearrangements, especially inversions, were pervasive between these two genomes. For example, two large inversions $(\sim 11.8 \mathrm{Mb}$ and $\sim 8.9 \mathrm{Mb}$ ) were identified on the two homologous chromosomes of Longmi4 (Pm5 and Pm8) that supported by intact scaffolds (Fig. $1 \mathrm{~b}$ and Supplementary Fig. 4). Besides, we also identified an interchromosomal exchange which was estimated to be occurred before the tetraploidization event, as reflected by a quartet among the chromosome ends of Pm3, Pm10, Pm14, and Pm15.

Gene annotation and gene family analysis. To annotate the protein-coding genes in broomcorn millet, we generated RNAseq data from the aerial part of Longmi4 seedlings and collected public available RNA-seq data of broomcorn millet, which covered the major developmental stages and tissues with a total data volume of $\sim 68.6 \mathrm{~Gb}$ (Supplementary Table 3 ). We also used the protein sequences from other related plant species, including $S$.

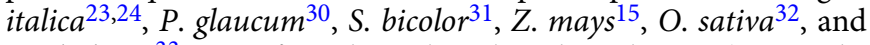
A. thalian $a^{33}$ to perform homology-based prediction (see Methods). In total, we predicted 63,671 genes in broomcorn millet, which was nearly two times the number of genes in S. italica reference genome Yugul (34,584 genes, v2.2 $)^{23}$. There were 62,934 genes $(\sim 98.8 \%)$ that could be assigned to 18 pseudomolecules, with the gene density highly skewed toward the distal ends of chromosome arms (Supplementary Fig. 5). The average length of transcripts $(\sim 2883 \mathrm{bp})$, coding regions $(\sim 1023 \mathrm{bp})$, and introns $(\sim 1270 \mathrm{bp})$ in broomcorn millet were highly similar with other important cereal crops (Supplementary Table 6). As a consequence of WGD, the percentage of WGD or segmental duplicated genes in broomcorn millet (39,769 genes, $63.2 \%)$ was substantially higher than foxtail millet (5805 genes, $16.9 \%$ ). On the contrary, only 5248 genes $(\sim 8.3 \%)$ in broomcorn millet were identified as singletons, which were much lower than that in foxtail millet (7367 genes, $21.5 \%$, Supplementary Table 7).

Using foxtail millet as a representative of the genome organization of two ancestral diploid genomes in broomcorn millet, we are able to study the gene loss and retentions following tetraploidization in broomcorn millet ${ }^{29}$. We identified 19,609 genes in foxtail millet ( $56.7 \%$ of total genes in Yugu1) that were syntenic with at least one subgenome in broomcorn millet (Supplementary Table 8). In consistent with a nearly double number of genes in broomcorn millet $(63,671)$ compared with foxtail millet $(34,584)$, we found the majority $(16,884, \sim 86.2 \%)$ of syntenic genes in foxtail millet have two homologous copies retained in broomcorn millet (Fig. 2 and Supplementary Table 8). The remaining $2725(\sim 13.8 \%)$ genes in foxtail millet have only one syntenic homolog in broomcorn millet. It was contrasting with the drastic gene loss after WGD reported in maize ${ }^{29}$ and soybean $^{34}$, which may be due to the more recent WGD in broomcorn millet as compared with soybean and maize (discussed later). Also, the two subgenomes of broomcorn millet shown approximately the same level of gene retentions (Fig. 2), as opposed to the bias of gene fractionation in maize ${ }^{29}$ and Brassica rapa ${ }^{35}$.

We further annotated the genes in Longmi4 via the homology of functional domains from InterPro database ${ }^{36}$. In total, there were 62,270 genes $(\sim 97.8 \%)$ that could be annotated by InterProScan, among which 46,299 ( 72.7\%), 53,289 ( 83.7\%), $19,959(\sim 31.3 \%)$, and $41,091(\sim 64.5 \%)$ genes could be annotated by Pfam ${ }^{37}$, PANTHER $^{38}$, ProSite ${ }^{39}$, and Gene $3 \mathrm{D}^{40}$, respectively (Supplementary Fig. 6). We assigned Gene Ontology (GO) to 35,560 genes $(\sim 55.8 \%)$ which included biological process $(N=21,517)$, cellular component $(N=8216)$, and molecular functions $(N=31,667)$. We also identified 3273 transcription factors from 55 gene families in Longmi4, including the major families of MYB, bHLH, ERF, NAC, and bZIP which contained 366, 295, 293, 243, and 171 genes, respectively (Supplementary Table 9). 

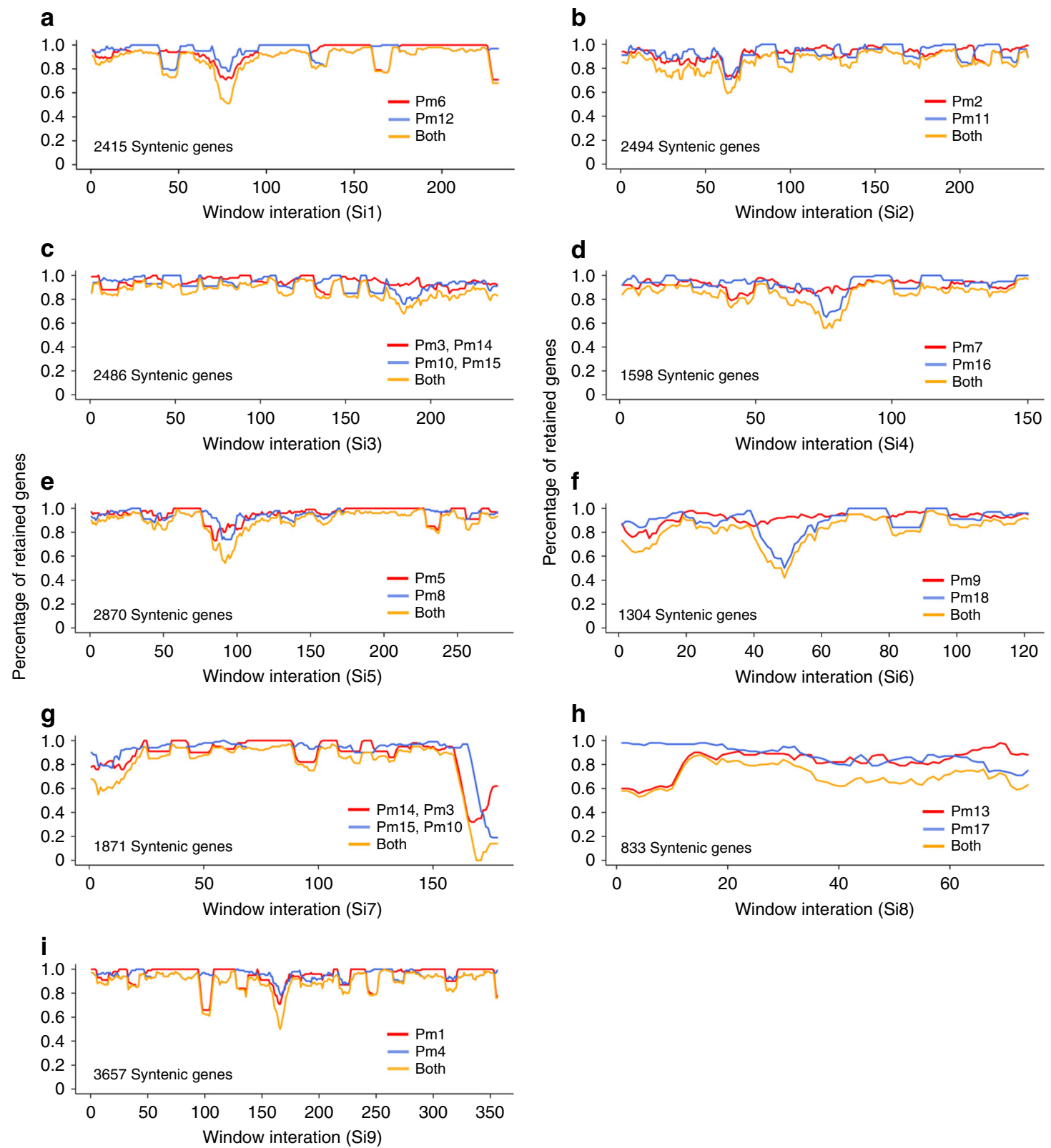

Fig. 2 Gene loss and retentions in broomcorn millet. a sliding window approach with window size of 100 syntenic genes and step size of 10 syntenic genes was used to show the percentage of retained genes in subgenome1 (red), subgenome2 (blue), and both (yellow) in broomcorn millet using foxtail millet (a i) as a reference. In total, there were 19,609 genes in foxtail millet that were syntenic with at least one subgenome in broomcorn millet, among which $16,884(\sim 86.2 \%)$ syntenic genes have two homologous copies retained in broomcorn millet. Source Data are provided as a Source Data file

To further reveal the lineage-specific expansion of genes families, we clustered the genes of broomcorn millet with foxtail millet, pearl millet, sorghum, and maize by OrthoMCL ${ }^{41}$, and identified 12,022, 11,686,10,776, 11,399, and 11,104 families for each species, respectively (Supplementary Fig. 7). As expected, within the majority $(7124, \sim 83.6 \%)$ of shared gene families (8517), the numbers of genes in broomcorn millet were higher than that in both foxtail millet and pearl millet. Furthermore, the numbers of lineage-specific gene families $(N=801$, Supplementary Fig. 7) and genes (6592) therein were both highest in broomcorn millet, some of which may be due to the neofunctionalization after $\mathrm{WGD}^{42}$. We annotated the lineagespecific genes in broomcorn millet and found that it contained a variety of functional domains, including Cytochrome P450, NBARC domains (also called NBS domains), sugar transporters, and wall-associated receptor kinase, etc. GO enrichment analysis of lineage-specific genes revealed 13 significant terms in molecular functions, mainly involved in hydrolase activities (Supplementary Fig. 8).

Mining the genome of broomcorn millet uncovered the genes potentially involved in both biotic and abiotic stress resistance in broomcorn millet. In total, we identified 493 genes containing NBARC domain (Pfam: PF00931) that may be involved in disease resistance ${ }^{43}$, of which 20 genes (seven gene families) were specific in broomcorn millet. In consistent with that in pearl millet ${ }^{30}$, the distribution of NB-ARC genes was highly biased in broomcorn millet, with gene clusters observed at chromosome ends of pm13 and pm17 (Supplementary Fig. 9). As a crop with extremely strong drought tolerance, we also identified 15 ABA or WDS (water-deficiency stress) responsive genes (Pfam: PF02496) ${ }^{44}$ in 
broomcorn millet. Interestingly, four of these ABA genes were constitutively expressed with relatively high expressional level across all the samples we examined, even for the tissues or stages without salt or drought treatment (Supplementary Fig. 10). Further experimental validations are needed to elucidate the functional role of these genes in the drought tolerance of broomcorn millet.

Recent bursts of Gypsy elements in broomcorn millet. By applying a de novo repeat family identification approach ${ }^{45}$, we totally identified $\sim 458.9 \mathrm{Mb}$ sequence as repeat elements in broomcorn millet, which constituted $\sim 54.1 \%$ of the genome. The percentage of repeat elements in broomcorn millet was between that of foxtail millet $(\sim 46.8 \%)$ and pearl millet $(\sim 68.0 \%$, Supplementary Table 10). For the DNA transposons, the proportion of broomcorn millet $(\sim 4.8 \%)$ was comparable with pearl millet $(\sim 4.7 \%)$ while much lower compared with foxtail millet $(\sim 10.2 \%)$, including the superfamilies of CMC-EnSpm, MULE-MuDR, and PIF-Harbinger. Considering the close relationship among these three species, we inferred that lineage-specific bursts of DNA transposons may have been happened in foxtail millet. Helitrons were relatively low in broomcorn millet $(\sim 0.44 \%)$, foxtail millet $(\sim 0.63 \%)$, and pearl millet $(\sim 0.11 \%)$, as compared with that in maize $(\sim 2.2 \%)^{46-48}$. We also found a relatively even distribution for both DNA transposons and Helitrons along the chromosomes, except several relative depletions around centromeric regions (as defined by gene density valleys in the middle of chromosomes, Supplementary Fig. 5).

Similar to other cereal crops ${ }^{23,30}$, LTR retrotransposon ( $37.1 \%)$, especially the Gypsy superfamily $(\sim 31.4 \%)$, constituted the majority of repeat elements in broomcorn millet. Gypsy elements were highly enriched around centromeric regions in both broomcorn millet and foxtail millet. While, the distribution of Copia elements was contrasting between these two species, especially around the centromeric regions (Supplementary Figure 5). There were also large differences of Gypsy-to-Copia ratio among the major crops in Paniceae, with the highest of $\sim 7.16$ in broomcorn millet, followed by foxtail millet ( 3.9), sorghum $(\sim 3.7)$, pearl millet $(\sim 2.24)$, and lowest in maize $(\sim 2.0)$. To explain these differences, we dated the activity of both Gypsy and Copia elements in these crops. We found very recent bursts of Gypsy elements in both maize and sorghum, followed by the bursts of other three species that were all within $\sim 1$ Mya (Fig. 3a). The amplifications of Copia elements were also very recent in foxtail millet, sorghum, and maize $(<1$ Mya), which was consistent with a previous estimation of LTR amplifications in these species 23,49 . While, the activity of Copia elements was relatively old in broomcorn millet ( $\sim 2$ Mya, Fig. $3 b)$, which may explain the extremely high Gypsy-to-Copia ratio in broomcorn millet, since no recent bursts of Copia elements were detected.

The phylogeny of Paniceae. Previous studies have established the phylogeny in the grass subfamily Panicoideae, although the genetic information may be limited to have an exact estimation of the evolutionary timeline ${ }^{23,50}$. Furthermore, as a model species in Paniceae, the timing of the tetraploidization in the lineage of broomcorn millet remained unresolved. By taking advantage of the high-quality assembly of broomcorn millet in this study, in combination with the newly published genomes of pearl millet ${ }^{30}$ and Dichanthelium oligosanthes ${ }^{51}$, we were able to reconstruct the phylogeny in Paniceae (Fig. 4). We firstly estimated the Ks (synonymous substitution rate) of orthologous gene pairs between broomcorn millet and foxtail millet, and the peak of $K s$ $(\sim 0.162)$ nearly coincided with the peak between sorghum and maize $(\sim 0.152)$, which was consistent with the inference that the lineages between broomcorn millet and foxtail millet diverged $\sim 13.1 \mathrm{Mya}^{23}$, slightly earlier than that between sorghum and maize ( 11.9 Mya) ${ }^{52}$. Phylogenetic data supported Dichanthelium as a distinct genus in Paniceae ${ }^{51}$, while the peak of $K s$ between Dichanthelium oligosanthes and foxtail millet $(\sim 0.177, \sim 14.3$ Mya) nearly colocalized with that between broomcorn millet and foxtail millet, indicating a close split of the progenitors among these three species (Fig. 4a). The divergency between foxtail millet and pearl millet was more recent, with the peak of $K s(\sim 0.121)$ corresponded to $\sim 9.81 \mathrm{Mya}$, slightly older than a previous estimation of $\sim 8.3 \mathrm{Mya}^{23}$. Finally, the Andropogoneae and Paniceae shared a common ancestor before $23.5 \mathrm{Mya}$, as revealed by the peak of $K s$ between foxtail millet and sorghum $(\sim 0.286)$ and that between broomcorn millet and sorghum $(\sim 0.295)$.

We next estimated the time of tetraploidization in the lineage of broomcorn millet by calculating the $K s$ between paralogous genes. We found two peaks for the Ks of broomcorn millet, with the secondary one $(\sim 1.08, \sim 85.9$ Mya) that far preceded the common ancestor between Andropogoneae and Paniceae, which may be originated from the WGD shared by all the grass as reported to be happened $\sim 70 \mathrm{Mya}^{24}$. We further confirmed this WGD in foxtail millet, since only a single peak of $K s$ was detected $(\sim 1.00$, Fig. $4 \mathrm{a})$ and no recent WGD happened in its lineage (Fig. 4b). We further estimated that the two ancestor genomes in broomcorn millet diverged $\sim 5.91$ Mya (Ks $\sim 0.073$ ), suggesting that the tetraploidization of broomcorn millet was more recent than that in soybean $(G \text {. soja, } \sim 13 \mathrm{Mya})^{34}$, probably also recent than maize $(\sim 5-12 \mathrm{Mya})^{52}$. In allotetraploid cottons $(G$. hirsutum), the divergency time between A- and D-progenitor genomes was estimated to be $\sim 6.0-6.3 \mathrm{Mya}$, and the allotetraploid was formed around 1-1.5 $\mathrm{Mya}^{53}$. In addition, gene loss in both $\mathrm{A}$ and $\mathrm{D}$ genomes of tetraploid cottons were rare $^{53}$. We thus hypothesized that the time of allotetraploid was more recent than $\sim 5.91$ Mya in broomcorn millet.

\section{Discussion}

We report here a high-quality reference genome of broomcorn millet. The quality of our assembly as reflected by the contig N50 $(\sim 2.55 \mathrm{Mb})$, scaffold $\mathrm{N} 50(\sim 8.24 \mathrm{Mb})$, or super scaffolds were considerably better than several other recently accomplished crop genome assemblies (such as rice ${ }^{12,13}$, maize ${ }^{14,15}$, quinoa ${ }^{19,20}$, barley $^{21}$, and durian ${ }^{18}$ ), which might be attributed to the unique combination of methods we used (deep PacBio sequencing, BioNano optical mapping, and in vivo $\mathrm{Hi}-\mathrm{C}$ scaffolding), in addition to the fact that the genome of broomcorn millet is highly homozygous. Recently, both the in vivo $\mathrm{Hi}-\mathrm{C}^{21}$ and in vitro Chicago technology ${ }^{18,19}$ have been shown to be able to order and orient the scaffolds into chromosomal level in several plant and animal species. We demonstrated here that a high-quality chromosome scale assembly could be generated through in vivo $\mathrm{Hi}-\mathrm{C}$ mapping in a rather complex plant genome. Since in vivo $\mathrm{Hi}-\mathrm{C}$ could also provide extra information of high-order chromatin architecture, it may potentially become more attractive as compared with the in vitro Chicago technology.

Unlike maize which experienced strong genome rearrangements and expansions after tetraploidization ${ }^{52}$, broomcorn millet retained the majority of two copies of ancestral genes, kept the basal chromosome numbers $(2 \mathrm{n}=4 \times=36)$ and experienced relatively weak genome expansion (as reflected by a nearly doubled genome size compared with foxtail millet), likely resulted from its more recent tetraploidization (within $~ 5.91$ million years). No biased fractionation of duplicated genes was detected between two subgenomes of broomcorn millet, as opposed to the strong fractionation in maize that subgenomel consistently retained more genes than subgenome $2^{29}$. It was also interesting 

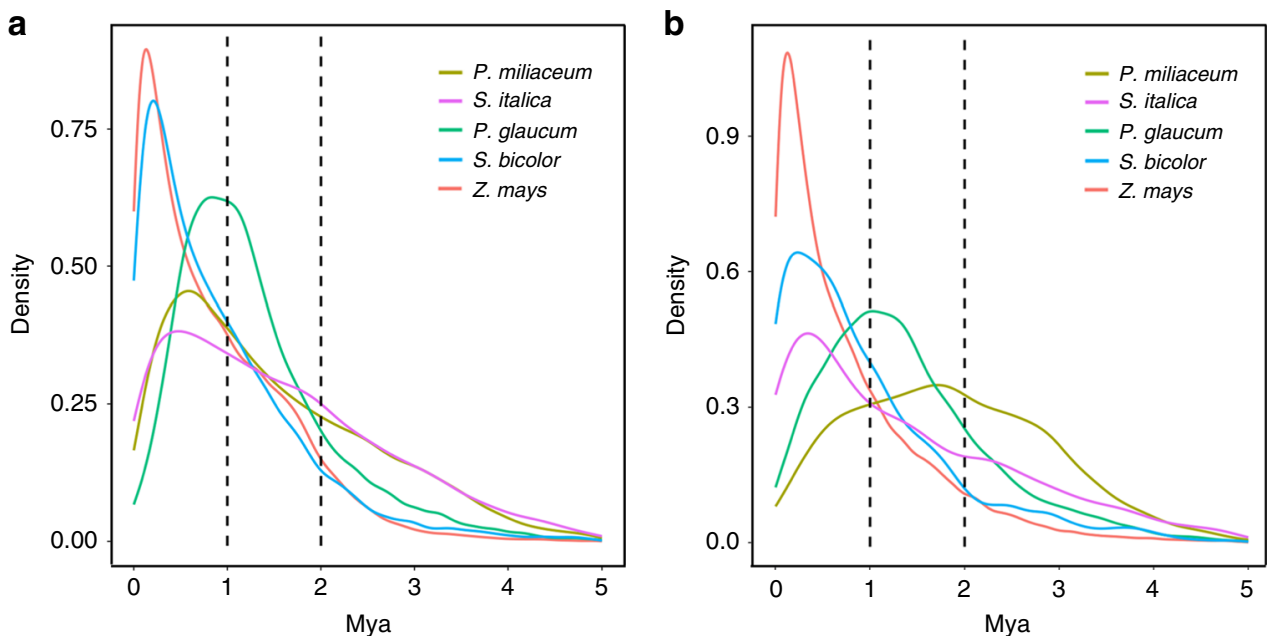

Fig. 3 The activity of LTR retrotransposons in Paniceae. The insertion time of Gypsy (a) and Copia (b) superfamilies were calculated in broomcorn millet (P. miliaceum), foxtail millet (S. italica), pearl millet (P. glaucum), sorghum (S. bicolor), and maize (Z. mays), respectively

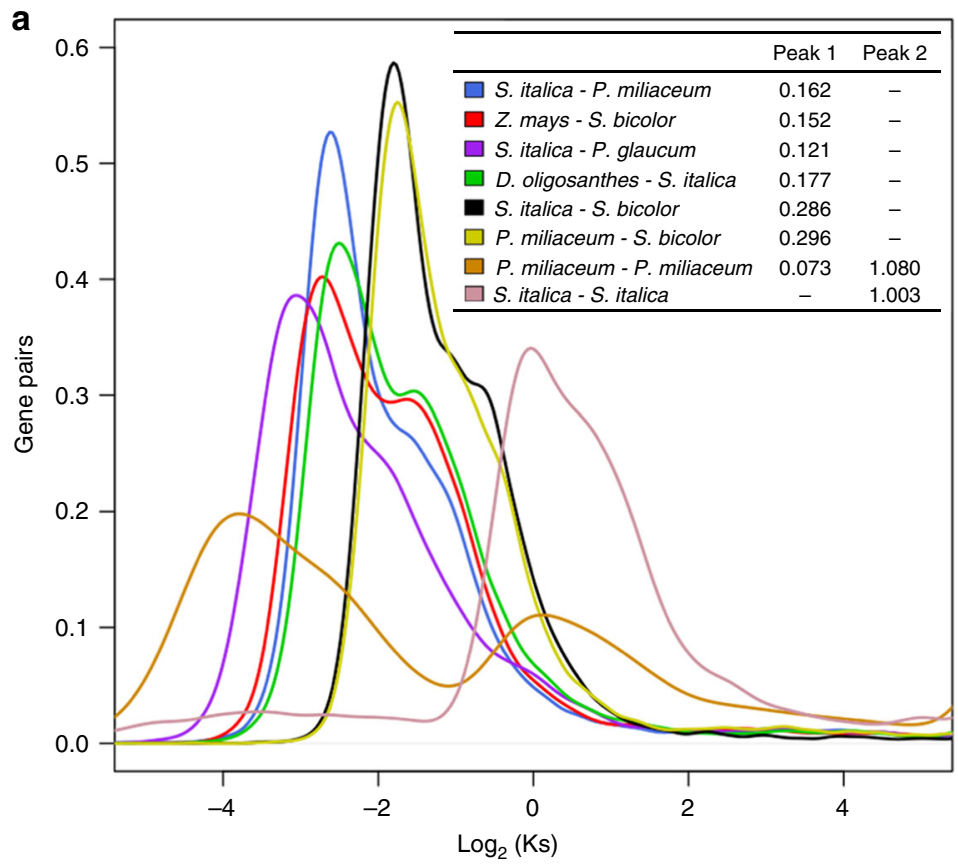

d

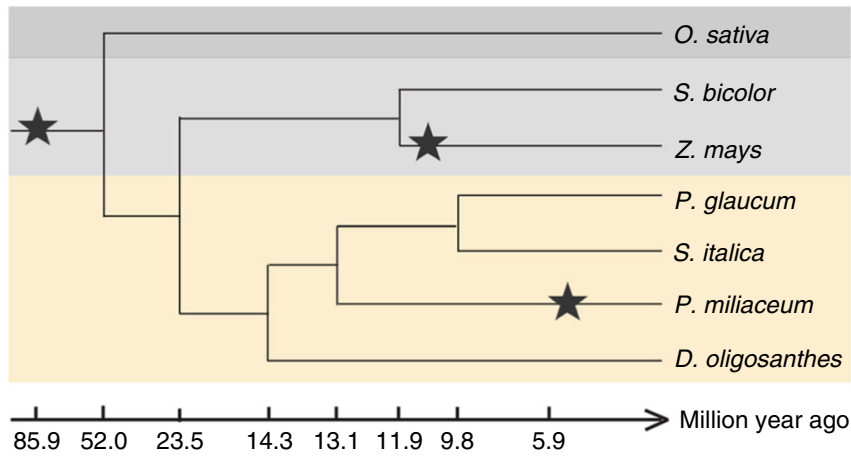

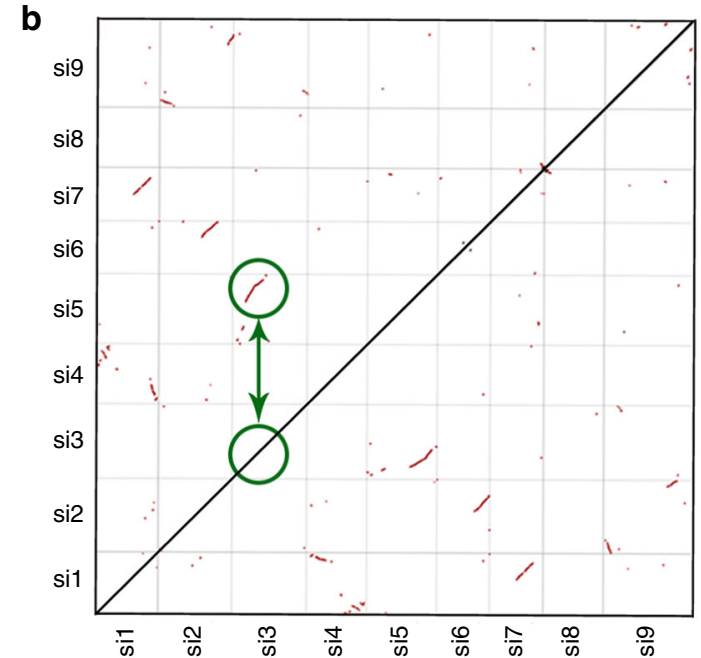

C

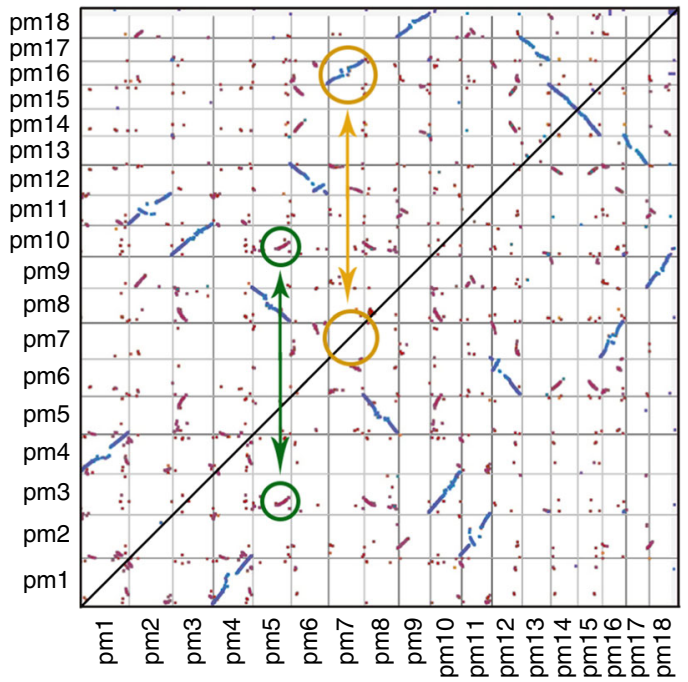

Fig. 4 The phylogeny of Paniceae. a Ks distribution of orthologous and paralogous genes in Paniceae. We further confirmed two WGD events in foxtail millet (b) and broomcorn millet (c), with the paralogous genes in green circles referred to the WGD shared by all grass, and the paralogous genes in orange circles referred to lineage-specific tetraploidization in broomcorn millet. d The phylogeny of Paniceae inferred from Ks distributions. The timeline was calculated based on the divergence between sorghum and maize (Ks 0.152, 11.9 Mya). The WGD events were marked by stars. Source Data of Fig. 4a are provided as a Source Data file 
that the time between $\sim 12$ and $~ 15$ Mya was an important period during the evolution of Paniceae, since a close split of the progenitors of broomcorn millet, foxtail millet, and Dichanthelium oligosanthes happened at that time interval (as well as that between sorghum and maize).

The availability of this genome will no doubt facilitate the comparative genomic researches between Panicum and other crops. Interestingly, as a tetraploid crop, the biomass of broomcorn millet is unexpectedly low, especially when compared with its close relative energy crop switchgrass. It was inferred that two independent tetraploidization events happened in these two close relatives $^{23}$. Further comparative genomic analysis will eventually uncover the genetic basis of phenotype difference between these two closely related tetraploid species in the future.

\section{Methods}

Plant materials. The seeds of Longmi4 were grown at dark conditions under $25^{\circ} \mathrm{C}$ after sowing for 14 days, then the aerial parts of seedlings were harvested and mixed, frozen immediately in liquid nitrogen for the extraction of genomic DNA. High molecular genomic DNA was extracted from isolated nuclei for sequencing library construction.

PacBio and Illumina sequencing. Libraries for single molecule real-time (SMRT) PacBio genome sequencing were constructed following the standard protocols of Pacific Biosciences company. Briefly, high molecule genomic DNA was sheared to $\sim 20 \mathrm{~kb}$ targeted size, followed by damage repair and end repair, blunt-end adaptor ligation, and size selection. Finally, the libraries were sequenced on the PacBio Sequel platforms.

The Illumina libraries were constructed according to the standard manufacturer's protocol (Illumina). Briefly, around $5 \mu \mathrm{g}$ DNA was fragmented and followed by size selection ( $450 \mathrm{bp}$ ) by agarose gel electrophoresis. The ends of selected DNA fragments were blunted with an A base overhang and ligated to sequencing adapters. All the libraries were sequenced on Illumina X-ten platform with pair-end sequencing strategy.

Genome survey. K-mer distribution was estimated by using jellyfish (http://www. genome.umd.edu/jellyfish.html, v2.2.6) with parameters -m 17 -s $200 \mathrm{M}$-C. The heterozygosity ratio was estimated by the online tool of GenomeScope (http://qb. cshl.edu/genomescope/). Finally, the genome size was calculated according to the formula that Genome_Size = K-mer coverage/Mean k-mer depth.

De novo assembly and polish of the genome. The raw contigs were assembled by Falcon (https://github.com/PacificBiosciences/falcon, v1.8.7 ${ }^{25}$ with the following steps: (a) raw reads overlapping for error correction; (b) pre-assembly and error correction; (c) overlap detection and filtering; (d) graph construction and contigs generation. The parameters during Falcon assembly were listed as follows:

length_cutoff $=11 \mathrm{~Kb}$, length_cutoff_pr $=15 \mathrm{~Kb}$, pa_HPCdaligner_option $=-\mathrm{v}$ -B128 -M24 -t12 -e 0.75 -k18 -w8 -h180 -T32 -12800 -s1000, ovlp_HPCdaligner_option $=-\mathrm{v}-\mathrm{B} 128-\mathrm{t} 12$-h280 -e $0.96-\mathrm{k} 22$-T32 -13200 -s1000. Then, the PacBio reads were mapped back to the raw contigs by Blasr (https://github.com/ PacificBiosciences/blasr, v5.1) ${ }^{54}$ with parameters (--bam --bestn 5 --minMatch 18 --nproc 4 --minSubreadLength 1000 --minAlnLength 500 --minPctSimilarity 70 --minPctAccuracy 70 --hitPolicy randombest --randomSeed 1) and contigs were further corrected by Arrow (https://github.com/PacificBiosciences/

GenomicConsensus, v2.1.0) with the parameter -j 30. Finally, Illumina reads were mapped back to the improved contigs and corrected by Pilon (https://github.com/ broadinstitute/pilon, v1.20 $)^{55}$ to generate the final consensus contigs with parameters --genome reference.fasta --changes --vcf --diploid --fix bases --threads 40 --mindepth 20.

BioNano optical maps and scaffold construction. High molecular genomic DNA were digested with single-stranded nicking endonuclease Nt. BspQI, then labeled using the IrysPrep Labelling mix and Taq polymerase according to standard BioNano protocols. Labeled DNA was imaged with BioNano Irys system, and raw BioNano data were assembled into optical map by IrysSolve (https:// bionanogenomics.com/support/software-downloads/, BioNano Genomics) with default parameters. Next, the optical map was aligned to PacBio contigs by IrysSolve, resolved the conflicts, and built the scaffolds according to the overlapping information between contigs and optimal maps.

Hi-C library constructions. The aerial parts of Longmi4 seedlings (14 days) were harvested and crosslinked by $40 \mathrm{ml} 2 \%$ formaldehyde solution at room temperature for $15 \mathrm{~min}$. Then, a total of $4.324 \mathrm{ml}$ of $2.5 \mathrm{M}$ glycine was added to quench the cross-linking reaction. The supernatant was removed and tissues were ground with liquid nitrogen and resuspended with $25 \mathrm{ml}$ of extraction buffer I (0.4 M sucrose, $10 \mathrm{mM}$ Tris- $\mathrm{HCl}, \mathrm{pH} 8.0,10 \mathrm{mM} \mathrm{MgCl}_{2}, 5 \mathrm{mM} \beta$ mercaptoethanol, $0.1 \mathrm{mM}$ phenylmethylsulfonyl fluoride [PMSF], and 1x protease inhibitor, Roche), then filtered through miracloth (Calbiochem). The filtrate was centrifuged at $3500 \mathrm{~g}$ at $4^{\circ} \mathrm{C}$ for $20 \mathrm{~min}$. The pellet was resuspended in 1 $\mathrm{ml}$ of extraction II $(0.25 \mathrm{M}$ sucrose, $10 \mathrm{mM}$ Tris- $\mathrm{HCl}, \mathrm{pH} 8,10 \mathrm{mM} \mathrm{MgCl}, 1 \%$ Triton X-100, $5 \mathrm{mM} \beta$-mercaptoethanol, $0.1 \mathrm{mM}$ PMSF, and $1 \mathrm{x}$ protease inhibitor) and centrifuged at $18,400 \mathrm{~g}$ and $4{ }^{\circ} \mathrm{C}$ for $10 \mathrm{~min}$. The pellet was resuspended in $300 \mu \mathrm{l}$ of extraction buffer III $(1.7 \mathrm{M}$ sucrose, $10 \mathrm{mM}$ Tris- $\mathrm{HCl}, \mathrm{pH} 8$, $0.15 \%$ Triton X-100, $2 \mathrm{mM} \mathrm{MgCl}_{2}, 5 \mathrm{mM} \beta$-mercaptoethanol, $0.1 \mathrm{mM}$ PMSF, and $1 \mathrm{x}$ protease inhibitor) and loaded on top of an equal amount of clean extraction buffer III, then centrifuged at $18,400 \mathrm{~g}$ for $10 \mathrm{~min}$. Discard the supernatant and then wash the pellet twice by resuspending it in $500 \mu \mathrm{l}$ ice cold $1 \mathrm{x}$ CutSmart buffer and then centrifuging the sample for $5 \mathrm{~min}$ at $2500 \mathrm{~g}$. The nuclei were washed by $0.5 \mathrm{ml}$ of $1 \mathrm{x}$ restriction enzyme buffer and transferred to a safe-lock tube. Next, the chromatin is solubilized with dilute SDS and incubation at $65^{\circ} \mathrm{C}$ for $10 \mathrm{~min}$. After quenching the SDS by Triton X-100, overnight digestion was applied with 4 bp cutter restriction enzyme ( 400 units MboI) at 37 ${ }^{\circ} \mathrm{C}$ on rocking platform. The next steps are $\mathrm{Hi}-\mathrm{C}$-specific, including marking the DNA ends with biotin-14-dCTP and performing blunt-end ligation of crosslinked fragments. The proximal chromatin DNA was re-ligated by ligation enzyme. The nuclear complexes were reversed crosslinked by incubating with proteinase $\mathrm{K}$ at $65^{\circ} \mathrm{C}$. DNA was purified by phenol-chloroform extraction. Biotin-C was removed from non-ligated fragment ends using T4 DNA polymerase. Fragments were sheared to a size of $100-500$ base pairs by sonication. The fragment ends were repaired by the mixture of T4 DNA polymerase, T4 polynucleotide kinase, and Klenow DNA polymerase. Biotin-labeled Hi-C sample were specifically enriched using streptavidin magnetic beads. The fragment ends were adding A-tailing by Klenow (exo-) and then adding Illumina paired-end sequencing adapter by ligation mix. Finally, the $\mathrm{Hi}-\mathrm{C}$ libraries were amplified by $10-12$ cycles PCR, and sequenced in Illumina HiSeq instrument with $2 \times 150$ bp reads.

Pseudomolecule construction by Hi-C. Clean $\mathrm{Hi}-\mathrm{C}$ reads were aligned to scaffolds using bowtie2 (v2.0.5 $)^{56}$ with end-to-end model. Totally, we generated $\sim 622.2$ million pair-end reads and $\sim 115.6$ million were uniquely aligned to the scaffolds (Supplementary Table 3 ). Reads with low mapping quality $(\mathrm{maq}<20)$, multiple hits, duplications, and singletons were discarded. Then, HiC-Pro (https://github. com/nservant/HiC-Pro, v2.7.8 $)^{57}$ was used to detect the ligation site using an exact matching procedure and to align the $5^{\prime}$ end of reads back to the scaffolds. There were $\sim 64.9$ million valid interaction pairs that were used to build the interaction matrices and draw the heatmap with Juicebox (https://github.com/aidenlab/ Juicebox, v1.8.8) software ${ }^{58}$. We then used Lachesis (https://github.com/ shendurelab/LACHESIS) ${ }^{27}$ to cluster, order, and orient the scaffolds. First, we clustered 495 scaffolds $(839,447,339 \mathrm{bp}, \sim 98.95 \%)$ into 18 chromosome groups according to the agglomerative hierarchical clustering algorithm. Within each cluster, a minimum spanning tree is found and the longest path in the tree is extracted as the trunk, an incomplete but high-confidence ordering of scaffolds within each chromosome group ${ }^{27}$. There were 170 scaffolds that were ordered as trunks, which constituted $808,559,470$ bp $(\sim 95.3 \%)$ of the total scaffolds. Scaffolds that were excluded from the trunks were reinserted between the trunks that maximized the amount of linkage between adjacent scaffolds, which resulted in 444 scaffolds that can be ordered $(838,831,330 \mathrm{bp}, \sim 98.9 \%)$. Finally, for each chromosome cluster, we ordered and traversed all the direction of the scaffolds through a weighted directed acyclic graph (WDGA) to predict orientation of each scaffold.

Gene annotation and gene family analysis-. To annotate the protein-coding genes in broomcorn millet, we combined three approaches: ab initio prediction, RNA-seq, and protein homology-based predictions. For the protein homologybased prediction, we downloaded the protein sequences of $S$. italica, $P$. glaucum, $S$. bicolor, Z. mays, O. sativa, and A. thaliana from Phytozome (http://www. phytozome.net), then aligned to the assembled scaffolds with TBLASTN (e-value $<$ 1e-5). Alignments within $20 \mathrm{~kb}$ were merged, and the alignments with coverage $>85 \%$ and identity $>75 \%$ were remained. Next, Genewise was used to annotate the gene models according to the alignments. For the RNA-seq based prediction, we generated RNA-seq data for the aerial parts of seedlings of Longmi4 grown at $25^{\circ} \mathrm{C}$ after sowing for 14 days, combined with the publicly available RNA-seq data (NCBI SRA accessions: ERR2040773, SRR1697309, SRR1697310,

SRR2179899 SRR2179908, SRR2179952, SRR2179961, SRR4069168 SRR4069173, Supplementary Table 2). All the RNA-seq data were aligned to scaffolds with Tophat $2^{59}$, and transcripts were further assembled by Cufflinks ${ }^{60}$ and StringTie ${ }^{61}$ Transdecoder was then used to predict the ORFs, and ORFs with length shorter than $600 \mathrm{bp}$ were filtered if no protein homology information (from TBLASTN alignments) or $\mathrm{Pfam}^{37}$ domains from $\operatorname{HMMer}^{62}$ (e-value $\left.<1 \mathrm{e}-5\right)$ could support this ORF. For the ab initio prediction, the repeat sequence in scaffolds were firstly masked by Repeatmasker (http://repeatmasker.org/, open-4.0.7), then the repeat masked scaffolds were annotated by Fgenesh ${ }^{63}$. The genes models from ab initio prediction will be retained if: (a) the predicted protein sequence could be aligned to the protein database above by BLASTP; (b) the predicted protein sequence could be supported by Pfam ${ }^{37}$ database $(e$-value $<1 \mathrm{e}-5) ;(\mathrm{c})$ more than 100 RNA-seq reads 
covered the coding region of the ab initio gene models. Finally, considering a better prediction of ORF structure for RNA-seq and homology-based predictions, we merged the gene models with the priority of RNA-seq-based > homology-based > $\mathrm{ab}$ initio with in-house Perl scripts. Potential transposons were further removed according to the protein annotation from InterProScan, which finally resulted in 63,671 protein-coding genes in Longmi4.

We used InterProScan (http://www.ebi.ac.uk/interpro/download.html, v5.15$54.0)$ to annotate the functional domains of genes with parameters $-\mathrm{f}$ tsv -goterms -iprlookup - $\mathrm{dp}-\mathrm{T}$. The transcription factors genes were annotated by the PlantTFDB (v4.0, http://planttfdb.cbi.pku.edu.cn/) ${ }^{64}$. GO enrichment analysis was performed by AgriGO (version 2, http://systemsbiology.cau.edu.cn/agriGOv2/). To cluster the genes into gene families, we aligned the representative protein sequence of each gene from broomcorn millet, foxtail millet, pearl millet, sorghum, and maize together by BLASTP $(e$-value $<1 e-5)$, then clustered the genes by OrthoMCL (http://orthomcl.org/orthomcl/ $/)^{41}$ with $e$-value cutoff of -20 and percent match cutoff of 50 .

Analysis of gene loss and retentions. To identify the duplicated genes in broomcorn millet, we aligned the representative protein of each gene from Longmi4 by blastp (-e 1e-10 -b 5 -v 5 -m 8 -o -a 8), then classified the type of duplicated genes by MCScanX (http://chibba.pgml.uga.edu/mcscan2/) with default parameters. To analyze the extent of gene loss and retentions in broomcorn millet, we used Coge pipeline (https://genomevolution.org/CoGe/) and blast the CDS of broomcorn millet against foxtail millet by Last, then used DAGchainer and QuotaAlign to find and merge syntenic blocks. Fractionation analysis was further applied by setting the syntenic depth to 2-to-1 between broomcorn millet and foxtail millet. A sliding window approach with window size of 100 syntenic genes and step size of 10 genes were used to show the proportion of retained genes in broomcorn millet.

Identification of repeat elements. We used a de novo repeat identification approach to annotate the repeat elements in broomcorn millet, foxtail millet ${ }^{23}$, and pearl millet ${ }^{30}$. First, we used RepeatModeler (open-1.0.11) to train a repeat database by the NCBI blast approach (-engine ncbi), then annotated the repeat elements according to the build database above by RepeatMasker (http:// repeatmasker.org/, open-4.0.7). To more accurately identity the LTR retrotransposons, we used LTRharvest ${ }^{65}$ (http://genometools.org/, v1.5.9) to identify the candidate LTRs with the parameters: - $\mathrm{v}$-mintsd 5 -maxtsd 20, then annotated the inner proteins of LTRs by LTRdigest with the parameters: -pptlen 1030 -pbsoffset 03 -trnas -hmms. Candidate LTRs that were classified into Gypsy and Copia superfamilies were processed into activity analysis. We extracted sequences of the long terminal repeats for each LTR, aligned them with MUSCLE (v3.8.31), then calculate the distance $K$ with Kimura Two-Parameter approach between LTRs by distmat in EMBOSS (v6.6.0). The activity of each LTR was calculated by the formular: $T=K /(2 \times r)$, where $r$ refers to a general substitution rate of $1.3 \times 10^{-8}$ per site per year in grass family ${ }^{66}$.

Phylogeny of broomcorn millet. We used the Coge pipeline (https:// genomevolution.org/coge/) to perform the comparative genomic analysis between species in Paniceae. Briefly, we used Last to blast the CDS against others, then used DAGchainer and QuotaAlign to find and merge syntenic blocks. We set the expected syntenic depth (1-to-1 or 2-to-1) to filter redundancy, then calculated the $K s$ rate between orthologous genes by CodeML. The Ks of paralogous genes were calculated by a similar approach. The time of the common ancestor between species was inferred according to the Ks of $\sim 0.152$ between maize and sorghum, since maize and sorghum were inferred to have a common ancestor before $~ 11.9$ $\mathrm{Mya}^{52}$.

Code availability. All the custom codes in this study were available upon request or can be downloaded from GitHub (https://github.com/caulai/broomcorn-milletgenome-assembly).

\section{Data availability}

Genome assembly was deposited into NCBI Genbank with accession ID PPDP00000000. The genome assembly and annotations have also been deposited in the Genome Warehouse in BIG Data Center, Beijing Institute of Genomics (BIG), Chinese Academy of Sciences, under accession number GWHAAEZ00000000. The genome sequence and gene annotations could also be found at Coge with genome ID 50980. The transcriptome data, Illumina reads (resequencing and $\mathrm{Hi}-\mathrm{C}$ ) and PacBio bam files generated in this study were deposited into NCBI SRA with accession number SRP128667. The source data underlying Figs. 1b, 2, 4a, and Supplementary Figs 2, 6 are provided as a Source Data file. A reporting summary for this Article is available as a Supplementary Information file.
Received: 18 June 2018 Accepted: 4 December 2018

Published online: 25 January 2019

\section{References}

1. Belton, P. S. \& Taylor, J. R. Sorghum and millets: protein sources for Africa. Trends Food Sci. \& Technol. 15, 94-98 (2004).

2. Lu, H. et al. Earliest domestication of common millet (Panicum miliaceum) in East Asia extended to 10,000 years ago. Proc. Natl. Acad. Sci. 106, 7367-7372 (2009).

3. Hunt, H. V. et al. Reticulate evolution in Panicum (Poaceae): the origin of tetraploid broomcorn millet, P. miliaceum. J. Exp. Bot. 65, 3165-3175 (2014).

4. Hunt, H. V. et al. Genetic diversity and phylogeography of broomcorn millet (Panicum miliaceum L.) across Eurasia. Mol. Ecol. 20, 4756-4771 (2011).

5. Ott, A. et al. Linked read technology for assembling large complex and polyploid genomes. BMC Genom. 19, 651 (2018).

6. Yue, H. et al. Transcriptome-wide identification and expression profiles of the WRKY transcription factor family in Broomcorn millet (Panicum miliaceum L.). BMC Genom. 17, 343 (2016).

7. Rajput, S. G., Santra, D. K. \& Schnable, J. Mapping QTLs for morpho-agronomic traits in proso millet (Panicum miliaceum L.). Mol. Breed. 36, 37 (2016).

8. Lu, F. et al. Switchgrass genomic diversity, ploidy, and evolution: novel insights from a network-based SNP discovery protocol. PLoS. Genet. 9, e1003215 (2013).

9. Bennetzen, J. L. \& Wang, H. The contributions of transposable elements to the structure, function, and evolution of plant genomes. Annu. Rev. Plant. Biol. 65, 505-530 (2014)

10. Adams, K. L. \& Wendel, J. F. Polyploidy and genome evolution in plants. Curr. Opin. Plant. Biol. 8, 135-141 (2005).

11. Edwards, D., Batley, J. \& Snowdon, R. J. Accessing complex crop genomes with next-generation sequencing. Theor. Appl. Genet. 126, 1-11 (2013).

12. $\mathrm{Du}, \mathrm{H}$. et al. Sequencing and de novo assembly of a near complete indica rice genome. Nat. Commun. 8, 15324 (2017).

13. Zhang, J. et al. Extensive sequence divergence between the reference genomes of two elite indica rice varieties Zhenshan 97 and Minghui 63. Proc. Natl. Acad. Sci. 113, E5163-E5171 (2016).

14. Sun, S. et al. Extensive intraspecific gene order and gene structural variations between Mo17 and other maize genomes. Nat. Genet. 50, 1289-1295 (2018).

15. Jiao, Y. et al. Improved maize reference genome with single-molecule technologies. Nature 546, 524-527 (2017).

16. Zhao, G. et al. The Aegilops tauschii genome reveals multiple impacts of transposons. Nat. Plants 3, 946-955 (2017).

17. Luo, M. et al. Genome sequence of the progenitor of the wheat D genome Aegilops tauschii. Nature 551, 498-502 (2017).

18. Teh, B. T. et al. The draft genome of tropical fruit durian (Durio zibethinus). Nat. Genet. 49, 1633 (2017).

19. Jarvis, D. E. et al. The genome of Chenopodium quinoa. Nature 542, 307-312 (2017).

20. Zou, C. et al. A high-quality genome assembly of quinoa provides insights into the molecular basis of salt bladder-based salinity tolerance and the exceptional nutritional value. Cell Res. 27, 1327 (2017).

21. Mascher, M. et al. A chromosome conformation capture ordered sequence of the barley genome. Nature 544, 427-433 (2017).

22. Vurture, G. W. et al. GenomeScope: fast reference-free genome profiling from short reads. Bioinformatics 33, 2202-2204 (2017).

23. Bennetzen, J. L. et al. Reference genome sequence of the model plant Setaria. Nat. Biotechnol. 30, 555-561 (2012).

24. Zhang, G. et al. Genome sequence of foxtail millet (Setaria italica) provides insights into grass evolution and biofuel potential. Nat. Biotechnol. 30 , 549-554 (2012).

25. Chin, C. et al. Phased diploid genome assembly with single-molecule real-time sequencing. Nat. Methods 13, 1050-1054 (2016).

26. Simão, F. A., Waterhouse, R. M., Ioannidis, P., Kriventseva, E. V. \& Zdobnov, E. M. BUSCO: assessing genome assembly and annotation completeness with single-copy orthologs. Bioinformatics 31, 3210-3212 (2015).

27. Burton, J. N. et al. Chromosome-scale scaffolding of de novo genome assemblies based on chromatin interactions. Nat. Biotechnol. 31, 1119 (2013).

28. Tiang, C., He, Y. \& Pawlowski, W. P. Chromosome organization and dynamics during interphase, mitosis, and meiosis in plants. Plant Physiol. 158 26 (2012).

29. Schnable, J. C., Springer, N. M. \& Freeling, M. Differentiation of the maize subgenomes by genome dominance and both ancient and ongoing gene loss. Proc. Natl. Acad. Sci. 108, 4069-4074 (2011).

30. Varshney, R. K. et al. Pearl millet genome sequence provides a resource to improve agronomic traits in arid environments. Nat. Biotechnol. 35, 969 (2017). 
31. Paterson, A. H. et al. The Sorghum bicolor genome and the diversification of grasses. Nature 457, 551-556 (2009).

32. Matsumoto, T. et al. The map-based sequence of the rice genome. Nature 436, 793-800 (2005).

33. Kaul, S. et al. Analysis of the genome sequence of the flowering plant Arabidopsis thaliana. Nature 408, 796-815 (2000).

34. Schmutz, J. et al. Genome sequence of the palaeopolyploid soybean. Nature 463, 178-183 (2010).

35. Wang, X. et al. The genome of the mesopolyploid crop species Brassica rapa. Nat. Genet. 43, 1035-1039 (2014).

36. Jones, P. et al. InterProScan 5: genome-scale protein function classification. Bioinformatics 30, 1236-1240 (2014).

37. Finn, R. D. et al. The Pfam protein families database: towards a more sustainable future. Nucleic Acids Res. 44, D279-D285 (2016)

38. Mi, H., Muruganujan, A., Casagrande, J. T. \& Thomas, P. D. Large-scale gene function analysis with the PANTHER classification system. Nat. Protoc. 8, 1551-1566 (2013).

39. Sigrist, C. J. et al. New and continuing developments at PROSITE. Nucleic Acids Res. 41, D344-D347 (2012).

40. Yeats, C. et al. Gene3D: modelling protein structure, function and evolution. Nucleic Acids Res. 34, D281-D284 (2006).

41. Li, L., Stoeckert, C. J. \& Roos, D. S. OrthoMCL: identification of ortholog groups for eukaryotic genomes. Genome Res. 13, 2178-2189 (2003).

42. Zhang, J. Evolution by gene duplication: an update. Trends Ecol. \& Evol. 18, 292-298 (2003).

43. McHale, L., Tan, X., Koehl, P. \& Michelmore, R. W. Plant NBS-LRR proteins: adaptable guards. Genome Biol. 7, 212 (2006).

44. Padmanabhan, V., Dias, D. M. \& Newton, R. J. Expression analysis of a gene family in loblolly pine (Pinus taeda L.) induced by water deficit stress. Plant Mol. Biol. 35, 801-807 (1997).

45. Smit, A. \& Hubley, R. RepeatModeler Open-1.0. Repeat Masker Website (2010).

46. Yang, L. \& Bennetzen, J. L. Distribution, diversity, evolution, and survival of Helitrons in the maize genome. Proc. Natl. Acad. Sci. 106, 19922-19927 (2009).

47. Du, C., Fefelova, N., Caronna, J., He, L. \& Dooner, H. K. The polychromatic Helitron landscape of the maize genome. Proc. Natl. Acad. Sci. 106, 19916-19921 (2009)

48. Schnable, P. S. et al. The B73 maize genome: complexity, diversity, and dynamics. Science 326, 1112-1115 (2009)

49. El Baidouri, M. \& Panaud, O. Comparative genomic paleontology across plant kingdom reveals the dynamics of TE-driven genome evolution. Genome Biol. Evol. 5, 954-965 (2013).

50. Kellogg, E. A., Aliscioni, S. S., Morrone, O., Pensiero, J. \& Zuloaga, F. A phylogeny of Setaria (Poaceae, Panicoideae, Paniceae) and related genera based on the chloroplast gene ndhF. Int. J. Plant Sci. 170, 117-131 (2009).

51. Studer, A. J. et al. The draft genome of the $\mathrm{C} 3$ panicoid grass species Dichanthelium oligosanthes. Genome Biol. 17, 223 (2016).

52. Swigoňová, Z. et al. Close split of sorghum and maize genome progenitors. Genome Res. 14, 1916-1923 (2004).

53. Zhang, T. et al. Sequencing of allotetraploid cotton (Gossypium hirsutum L. acc. TM-1) provides a resource for fiber improvement. Nat. Biotechnol. 33, 531 (2015).

54. Chaisson, M. J. \& Tesler, G. Mapping single molecule sequencing reads using basic local alignment with successive refinement (BLASR): application and theory. BMC Bioinforma. 13, 238 (2012).

55. Walker, B. J. et al. Pilon: an integrated tool for comprehensive microbial variant detection and genome assembly improvement. PLoS. One. 9, e112963 (2014).

56. Langmead, B. \& Salzberg, S. L. Fast gapped-read alignment with Bowtie 2. Nat. Methods 9, 357 (2012)

57. Servant, N. et al. HiC-Pro: an optimized and flexible pipeline for Hi-C data processing. Genome Biol. 16, 259 (2015).

58. Durand, N. C. et al. Juicebox Provides a Visualization System for Hi-C Contact Maps with Unlimited Zoom. Cell Syst. 3, 99-101 (2016).

59. Trapnell, C., Pachter, L. \& Salzberg, S. L. TopHat: discovering splice junctions with RNA-Seq. Bioinformatics 25, 1105-1111 (2009).
60. Trapnell, C. et al. Differential gene and transcript expression analysis of RNAseq experiments with TopHat and Cufflinks. Nat. Protoc. 7, 562-578 (2012).

61. Pertea, M. et al. StringTie enables improved reconstruction of a transcriptome from RNA-seq reads. Nat. Biotechnol. 33, 290-295 (2015).

62. Eddy, S. R. Accelerated profile HMM searches. LoS Comput. Biol. 7, e1002195 (2011).

63. Salamov, A. A. \& Solovyev, V. V. Ab initio gene finding in Drosophila genomic DNA. Genome Res. 10, 516-522 (2000).

64. Jin, J. et al. PlantTFDB 4.0: toward a central hub for transcription factors and regulatory interactions in plants. Nucleic Acids Res. 45, D1040-D1045 (2017).

65. Ellinghaus, D., Kurtz, S. \& Willhoeft, U. LTRharvest, an efficient and flexible software for de novo detection of LTR retrotransposons. BMC Bioinforma. 9 , 18 (2008).

66. SanMiguel, P., Gaut, B. S., Tikhonov, A., Nakajima, Y. \& Bennetzen, J. L. The paleontology of intergene retrotransposons of maize. Nat. Genet. 20, 43-45 (1998).

\section{Acknowledgements}

We thank the NextOmics (Wuhan, China) for their assistance to generate the PacBio data and genome assembly. We also thank BerryGenomics (Beijing, China) for their assistance in generation of the BioNano data. We appreciated Dr. Shi, Yiting from China Agricultural University for her helpful discussion with this manuscript. This work was supported by the National Key Research \& Development Program (2016YFD0101803, 2017YFD0101104, 2016YFD0100404), National Natural Science Foundation of China $(31421005,91635303)$ and 948 project $(2016-X 33)$.

\section{Author contributions}

J.S., W.S., and J.L. designed this research project. J.S., J.Z., M.L., X.G., P.H.L., L.W., P.L. H.Z., W.S., and J.L. collected the plant materials, performed the experiments, and generated the sequencing data. J.S. and X.M. assembled the contigs and scaffolds. J.S., W.Z. and L.H. analyzed Hi-C data. J.S. and Y.Z. annotated the genome. J.S. and X.M. performed comparative and evolutionary analysis. X.Z. and S.S. participated in the data analysis. J.S. and J.L. wrote the paper.

\section{Additional information}

Supplementary Information accompanies this paper at https://doi.org/10.1038/s41467018-07876-6.

Competing interests: The authors declare no competing interests.

Reprints and permission information is available online at http://npg.nature.com/ reprintsandpermissions/

Journal peer review information: Nature Communications thanks the anonymous reviewer(s) for their contribution to the peer review of this work. Peer reviewer reports are available

Publisher's note: Springer Nature remains neutral with regard to jurisdictional claims in published maps and institutional affiliations.

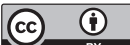

Open Access This article is licensed under a Creative Commons Attribution 4.0 International License, which permits use, sharing, adaptation, distribution and reproduction in any medium or format, as long as you give appropriate credit to the original author(s) and the source, provide a link to the Creative Commons license, and indicate if changes were made. The images or other third party material in this article are included in the article's Creative Commons license, unles indicated otherwise in a credit line to the material. If material is not included in the article's Creative Commons license and your intended use is not permitted by statutory regulation or exceeds the permitted use, you will need to obtain permission directly from the copyright holder. To view a copy of this license, visit http://creativecommons.org/ licenses/by/4.0/

(c) The Author(s) 2019 